\title{
Cost Comparison of an On-Premise IT Solution with a Cloud-Based Solution for Electronic Health Records in a Dental School Clinic
}

\begin{abstract}
Lynn Johnson, Cassandra Callaghan, Madhan Balasubramanian, Haris Haq, Heiko Spallek
Abstract: Electronic health records (EHRs) are increasingly moving towards cloud-based web environments. While cloud-based EHRs claim substantial benefits at reduced cost, little cost-benefit research exists for dental schools. The aim of this study was to examine the cost-benefits of a cloud-based EHR compared to an on-premise client-server EHR in the University of Michigan School of Dentistry (U-M Dent). Data were collected in 2016 from the U-M Dent cost-benefit comparison of tangible and intangible factors associated with implementing a new EHR, using the Total Cost of Ownership (TCO) framework from EDUCAUSE. The TCO framework assessed three factors: foundational (overarching aspects: three items), qualitative (intangibles: 56 items), and quantitative (actual costs). Stakeholders performed factor grading, and relative assessment scores were derived for each item as well as the overall factor. The cloud-based EHR solution received higher foundational and qualitative factor summary scores. The overall cost of an on-premise solution over a two-year period was approximately $\$ 2,000,000$ higher than a cloud-based solution. Cloud solutions did not carry any hidden costs, while such costs accounted for $8 \%(\sim \$ 540,000)$ of the overall costs of the on-premise solution. Across the two-year period, both one-time and ongoing costs were higher for the on-premise solution than the cloud-based solution (by $40.5 \%$ and $20.5 \%$, respectively). This study found that a cloud-based EHR system in the U-M Dent offered significant cost savings and unique benefits that were not available with the on-premise EHR solution. Based on cost, the U-M Dent has made a case for cloud-based EHR systems.
\end{abstract}

Lynn Johnson is Professor and Associate Dean, University of Michigan School of Dentistry; Cassandra Callaghan is Director of Dental Informatics, University of Michigan School of Dentistry; Madhan Balasubramanian is Sidney Sax National Health and Medical Research Council Research Fellow, University of Sydney, Australia and King's College London, United Kingdom; Haris Haq is a Consultant, Collaboration for Health IT; and Heiko Spallek is Professor, Head of School, and Dean, The University of Sydney School of Dentistry, Sydney, New South Wales, Australia. Direct correspondence to Dr. Lynn Johnson, University of Michigan School of Dentistry, 1011 North Michigan, Room 1216, Ann Arbor, MI 48130-1078; lynjohns@umich.edu.

Keywords: dental education, dental schools, dental school clinic, clinic management, electronic health records, cost-benefit analysis, organizational innovation

Submitted for publication 7/15/18; accepted 1/29/19; first published online 4/22/19

doi: 10.21815/JDE.019.089

$\mathrm{H}$ ealth care's most important information systems-electronic health records (EHRs) - are moving towards functioning in a cloud-based environment. To date, there is considerable evidence to suggest that cloud-based EHR systems offer substantial benefits when compared to on-premise client-server EHR systems that are more commonly used. ${ }^{1}$ The speed and flexibility of cloud resources can shorten the time for implementation, decrease burden on resources required for upgrades, and allow practitioners and patients to focus on a patient's health versus the technology, while concurrently allowing for improved cost benefits. ${ }^{2}$ As data for a cloud-based EHR are stored using external servers, they can be accessed through any device using an Internet connection. Cloud-based EHR systems provide better interoperability, scalability, maintainability, and accessibility and all at reduced costs when compared to on-premise solutions. ${ }^{3}$ Portability is vastly enhanced, as cloud systems enable easier movement of applications and data from one environment to another. These advantages are significant, and a cloud-computing framework can make patients' EHR sharing easier and allow health professionals to communicate better, as well as help lessen issues associated with on-premise systems such as network security and data storage. ${ }^{3,4}$ While there exist numerous and varied considerations in 
moving from traditional on-premise client-server EHRs to cloud-based EHRs, we found no research comparing them in a dental school environment.

Today, the bulk of North American dental schools operate in a fully digital format or are on track to do so within the next few years. ${ }^{5}$ In the U.S. alone, most dental schools have at least a traditional on-premise EHR. ${ }^{5,6}$ EHRs are said to be vital for patient safety and the maintenance of standards as they provide information for patient care, ensure fiscal accountability, track quality of patient care, manage clinic operations, and report student work. ${ }^{4}$ The adoption of EHRs in U.S. dental schools has been high, as opportunities to enhance clinical training, promote evidence-based practice, streamline billing and collections, and conduct research have interested academic dental institutions. $^{7}$

EHRs can help to reshape dental education and indeed the profession. ${ }^{8}$ They carry the potential to improve students' critical thinking skills and assist educators in better observing and grading dental students. ${ }^{9,10}$ The way students learn is being influenced as EHRs guide students in developing comprehensive patient notes and help them reflect on patient diagnosis before treatment. ${ }^{11,12}$ While engaging dental students in research undoubtedly broadens their horizons, ${ }^{13}$ it is hard to accomplish that if their career goal is to enter private practice. ${ }^{14,15}$ Enticing them by demonstrating the value of clinical data - data they will later collect themselves in their private practice-is one way EHRs can help design meaningful research experiences for dental students. Further, the leading professional dental organization in North America, the American Dental Association (ADA), has its own national standards on dental informatics (www.ada. org/en/science-research/dental-standards/standardscommittee-on-dental-informatics). Polverini and Krebsbach argued that when dentistry embraces a learning health care system, one can expect to realize an enhanced quality of oral health care, as well as increased efficiencies and better cost containment. ${ }^{10}$

The increased adoption of EHRs has revealed the benefits they bring to creating Learning Health Systems. Thus, the move towards cloud-based EHR solutions can be seen as a logical step for the future. The underlying assumption is that cloud solutions vastly enhance the benefits of using EHRs, at significant cost-savings. ${ }^{2,16}$ Nevertheless, no research exists on the costs and benefits of using a cloudbased system in dental school. Most dental schools do not track costs associated with the investment that has been made in their current health information technology (IT) infrastructure, let alone estimate the cost of future improvements. ${ }^{4}$ Without a cost-benefit comparison, the case for cloud-computing systems is diminished and, with that, all the tremendous benefits that such a solution brings. Therefore, the aim of this study was to examine the cost-benefits of a cloud-based EHR compared to an on-premise client-server EHR in the University of Michigan School of Dentistry.

\section{Methods}

All data for this study were made available from the University of Michigan School of Dentistry (U-M Dent), which is currently undergoing a transition from an on-premise client-server EHR system to a cloud-based EHR system. ${ }^{17}$ The data were collected in 2016 to gain an in-depth understanding of the risks, costs, and benefits of this transition. As an on-premise EHR system was already in place at the time of data collection, the study compared the costs of upgrading this on-premise system (to a new on-premise version) with the costs of deploying and maintaining a cloud-based EHR system. It needs to be stressed that this comparison is not a comparison of two different EHRs. It is a comparison of the cost of two different EHR technologies (on-premise and cloud-based). Thus, the names of the traditional on-premise vendor and cloud-based EHR service provider are not provided. (Readers may contact the corresponding author for information on the vendors or the protocols in place to preserve the confidentiality of vendor information used in this study.) Once an institution's leaders select the technology to use, they should select the EHR that best fits their institution.

\section{TCO Framework}

To conduct this cost-benefit analysis, we used a Total Cost of Ownership (TCO) framework provided by EDUCAUSE, an association and community of information technology leaders and professionals committed to advancing higher education. ${ }^{18}$ The TCO framework, developed by the EDUCAUSE Centre for Analysis and Research (ECAR), provides a holistic view on tangible and intangible values of implementing new IT solutions in higher education. ${ }^{2,19}$ More importantly, the framework offers a systematic approach for researchers to better examine the costs and benefits of an on-premise IT solution as compared to a cloud-based solution. ${ }^{2}$ 
The ECAR-TCO framework is comprised of three factors: foundational risks, qualitative factors, and quantitative factors. Foundational risks are overarching features that are essential by themselves and help determine the importance of other factors in the framework. For example, data sensitivity is a main foundational risk assessment necessary to identify the data being stored/processed and rank the sensitivity of the data relative to legal, regulatory, or policy requirements.

Qualitative factors are the intangible aspects that are not easily translated to financial terms but are still essential to gather a holistic view of the IT solutions being compared. These include factors such as agility (easy and quick deployment and subsequent changes), contract review (implementation with minimal contract review and negotiation), elasticity and scalability (easy and quick expansion during peak times and reduction in times of lower need), and security (effective mechanisms to monitor security events). The ECAR-TCO framework allows each institution to reflect its uniqueness through the assignment of importance to a factor. For U-M Dent, remote access was a strategic imperative and thus that factor was considered of high importance. For another institution, remote access might be of medium or low importance.

Quantitative factors include the costs involved in the deployment and maintenance of each IT solution in a higher education environment and could be readily identified by the financial/administrative team. These factors are grouped into three subcategories: hidden subsidies, one-time costs, and ongoing costs. Hidden subsidies include expenses that go unrecognized such as facilities (space in data center, offices, computers, etc.) and business continuity response (response to a disaster such as fire or earthquake). One-time costs are those that arise during implementation of a service and include expenses such as customization, system architecture, integration, migration, hardware, and software costs. Ongoing costs are cost factors that continue during the entire operation of the service such as subscription/licensing, data ingress/egress fees, and some labor costs. A summary and brief description of the factors that comprise the ECAR-TCO framework are shown in Table 1.

While the ECAR-TCO framework was built for understanding the costs and benefits of different IT solutions in a higher education environment by providing a list of factors/items, it also offers the flexibility for organizations to add to or modify the list of factors/items based on relevant needs. For this analysis, 56 additional qualitative items were identified as important. Four hidden/subsidies costs, ten one-time costs, and a further ten ongoing costs, along with three foundational items, were included in the grading and analysis. A more detailed description of all the factors can be obtained from the EDUCAUSE website. $^{2}$

\section{Factor Grading and Data Analysis}

Based on the ECAR-TCO framework, the dental informatics team in U-M Dent graded all factors (foundational, qualitative, and quantitative) by their level of importance into high, medium, and low. (The dental informatics team that conducted the review consisted of four personnel, the team director, the application/software lead, the server/ network lead, and the digital learning services lead. Key staff members were also consulted on assumptions and estimates.) While differences between the on-premise and cloud-based technologies prevent a complete "apples-to-apples" comparison of all factors, the team worked with clinical and financial experts as needed to equate all measures for the two systems. All work completed by the team was then carefully reviewed by a school leader outside the team (the director of billing and financial planning), who was tasked with challenging and verifying all estimates and projections. Lastly, the school leadership reviewed the TCO.

For each foundational and qualitative factor/ item, a relative assessment between the two IT EHR solutions (client-server on-premise and cloud-based) was performed, and a value of $+1,0$, or -1 was provided $(+1$ was more effective in mitigating risks and -1 was less effective). For the qualitative factors/ items, a similar relative assessment of the effectiveness of the two EHR solutions was performed, and a value of $+1,0$, or -1 was provided $(+1$ was more cost effective and -1 was less cost effective). To arrive at a numerical estimate for the foundational and qualitative factors, the levels of importance were given a score (high $=3$, medium $=2$, and low $=1$ ). These levels of importance scores were multiplied by the relative assessment of effectiveness scores $(+1,0$, or -1$)$ to arrive at overall summary scores.

The quantitative factors had measurable cost estimates. Costs were obtained for two years. (To maintain vendor confidentiality, the costs are presented as two-year costs and do not examine the years individually. For more information, contact the corresponding author.) The first year included 
Table 1. Summary of EDUCAUSE Centre for Analysis and Research Total Cost of Ownership (ECAR-TCO) framework

Factor

Foundational risks

Qualitative factors (ECAR-TCO framework includes these standard factors. U-M Dent added 56 factors that were important to its situation.)

Quantitative factors
Description

Overarching essential elements

- Data sensitivity: identify data to be stored and data sensitivity

- Business criticality: analyze business functions and their criticality

- Vendor capability: assess vendor's previous successes

Intangible but essential aspects

- Agility: speed of development and change

- Contract review and negotiation: amount of contract review and negotiation required

- Elasticity and scalability: easy and quick adjustment to needs

- Regulatory and policy requirements: level of compliance with external and internal regulations

- Security: ability to meet and monitor constantly escalating security threats

- Service levels: provide satisfactory service levels and guaranteed remedies

Hidden subsidies (one-time and ongoing)

- Facilities and energy: space in data center, offices, computers, etc. and cost of electricity to run infrastructure

- Indirect costs (grants): facilities and administrative costs to support related grants

- Disaster recovery system (readiness): costs for ongoing service to ensure system is available in event of a disaster

- Business continuity (response): costs associated with an actual incident

One-time costs (one-time implementation costs)

- Customization/business process reconfiguration: customize or configure tool to meet existing processes, or change existing process to match the tool

- System re-architecture: redesign system to meet needs

- Integration: connect with other systems

- Migration: training IT staff to support new system

- Hardware: servers, network, etc.

- Software/licensing: acquisition and associated support

- Training: faculty, students, and staff; rewriting documentation and training materials

- Staffing: temporary staff for moving to new system and shutting down current system

- Reporting: migrating or rewriting reports

- Remote and mobile access: an infrastructure that allows security access from an external location

Ongoing costs

- Subscription/licensing: cloud subscription fees or software licensing fees

- Software: other software

- Data ingress/egress: costs to upload or download data

- Service management staff: staff who manage operations of the service

- Database administrator staff: database administrator

- System administration staff: cloud or system administrator, data center staff, and network staff

- Security staff and related infrastructure: technical and physical security staff

- Technical support staff: help desk staff

- Functional staff: non-technical staff who perform the business functions

- Vendor/contract management staff: staff who manage relationship with the service provider/ vendor

UM-Dent=University of Michigan School of Dentistry

the actual costs of an upgrade of the on-premise EHR and the estimated implementation costs of the cloud EHR. The second year revealed the recurring costs of each system. For ease of calculation, salary and benefits were estimated at $\$ 100 \mathrm{~K} /$ year/person no matter what the role. Actual costs were available for hardware, network, and software expenses.
Estimated costs were calculated first by the dental informatics team and then verified by the school's director of billing and financial planning. Also, the upgrade of the on-premise EHR included upgrading the EHR software, infrastructure updates, and the reporting software. Rewriting the reports to work with upgraded reporting software was also included. 


\section{Results}

All three foundational risk items were ranked as having high importance, and the cloud-based EHR solution obtained a better overall summary score of 6 compared to a score of 3 for the on-premise EHR solution. Table 2 provides these summary scores for the foundational risk factors.

Regarding the qualitative assessment, 30 items were graded as having high importance, 21 as having medium importance, and five as having low importance. The cloud-based solution received a better overall summary score of 73 compared to a score of -40 for the traditional on-premise solution. Table 3 provides the summary scores for the qualitative factors. (For a detailed relative assessment of these qualitative factors, contact the corresponding author.)

The overall cost of upgrading and operating an on-premise EHR solution over a two-year period was

Table 2. High importance foundational risk factors and summary scores for cloud-based vs. on-premise electronic health record

\begin{tabular}{lcc} 
Factor & Cloud & On-Premise \\
\hline Data sensitivity & 3 & 0 \\
Business criticality & 3 & 3 \\
Vendor capability & 0 & 0 \\
Total & 6 & 3
\end{tabular}

Note: Scores show relative effectiveness as ranked -1 or 0 or +1 . Scores are multiplied by 3 as the level of importance for all factors was graded as high. approximately $\$ 2,000,000$ higher than the implementation and operation of a cloud-based EHR solution. While the cloud solution did not carry any associated hidden subsidies, those costs accounted for $8 \%$ (approximately $\$ 540,000$ ) of the overall costs of the traditional on-premise solution. These hidden subsidies for the on-premise solution included facilities, energy/electric costs $(\$ 240,000)$, disaster recovery systems $(\$ 200,000)$, and business continuity costs $(\$ 100,000)$. Across the two-year period, both one-time and ongoing costs were also higher for the on-premise solution than the cloud-based solution (by $40.5 \%$ and $20.5 \%$, respectively). The costs of a cloud-based EHR solution were higher than the on-premise solution mainly in subscription/licensing fees, migration costs, training (a new training program required development), and technical support. Figure 1 and Table 4 provide cost comparisons of the three subcomponents of the quantitative factors.

Table 3. Qualitative factors summary scores for cloudbased vs. on-premise electronic health record

\begin{tabular}{lcc} 
Factor & Cloud & On-Premise \\
\hline High importance & 51 & -33 \\
Medium importance & 12 & -6 \\
Low importance & -2 & 3 \\
Total & 61 & -36
\end{tabular}

Note: Scores reflect relative effectiveness: high importance items received a grade of 3 , medium importance a grade of 2 , and low importance a grade of 1 .

\section{Two year cost comparisons}

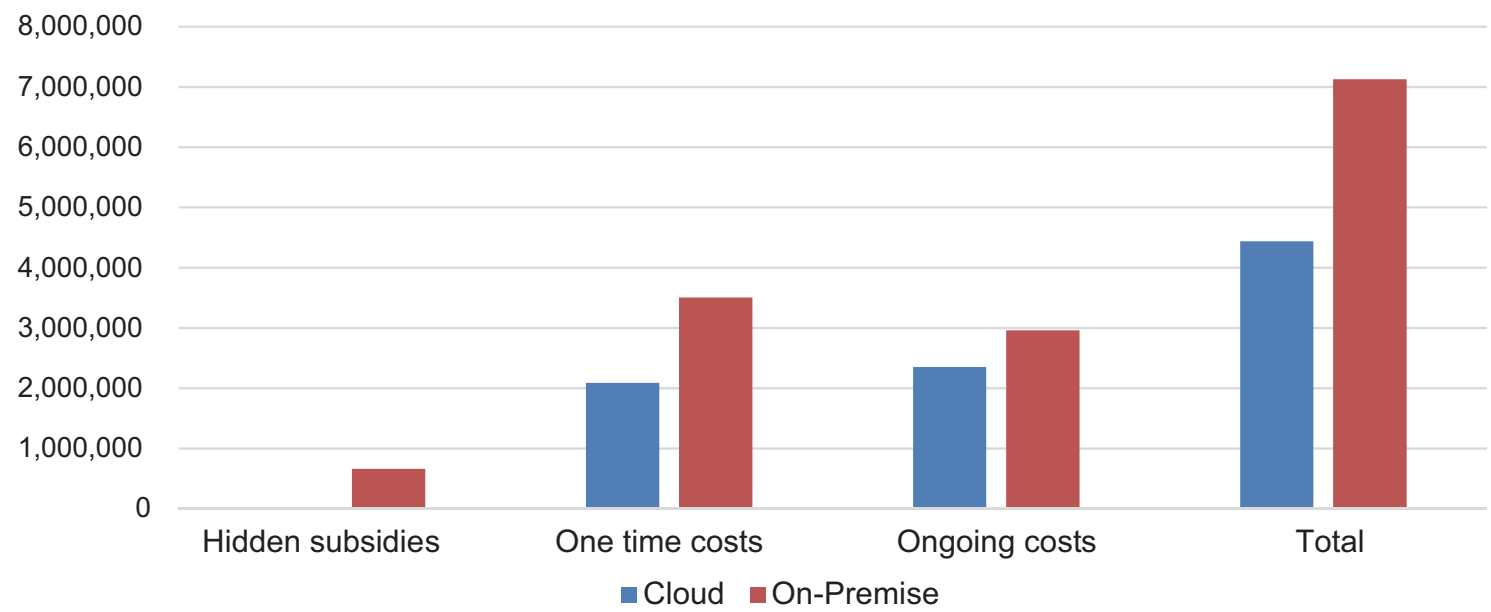

Figure 1. Summary of cost comparisons of cloud vs. on-premise electronic health record

Note: Estimates are in U.S. dollars. 


\begin{tabular}{|c|c|c|c|}
\hline Cost Type & Importance & Cloud & On-Premise \\
\hline \multicolumn{4}{|l|}{ Hidden subsidies } \\
\hline Facilities and energy & High & 0 & 239,908 \\
\hline Indirect costs (grants) & Low & 0 & 0 \\
\hline Disaster recovery system (readiness) & High & 0 & 200,000 \\
\hline Business continuity (response) & High & 0 & 100,000 \\
\hline \multicolumn{4}{|l|}{ One-time costs } \\
\hline Customization/business process reconfiguration & High & 0 & 150,000 \\
\hline System re-architecture & Medium & 0 & 100,000 \\
\hline Integration & High & 200,000 & 300,000 \\
\hline Migration & Low & 150,000 & 50,000 \\
\hline Hardware & High & 7,500 & 35,000 \\
\hline Software & Medium & 271,580 & 0 \\
\hline Training & Medium & 50,000 & 25,000 \\
\hline Staffing & Low & 890,000 & $1,383,760$ \\
\hline Reporting & Low & 696,800 & 930,280 \\
\hline Remote and mobile access & High & 0 & 550,000 \\
\hline \multicolumn{4}{|l|}{ Ongoing costs } \\
\hline Subscription/licensing & High & 346,991 & 217,767 \\
\hline Software & High & 0 & 0 \\
\hline Data ingress/egress & High & 0 & 0 \\
\hline Service management staff & Medium & 0 & 0 \\
\hline Application/database administrator staff & Medium & 0 & 50,000 \\
\hline System administration staff & High & 0 & 200,000 \\
\hline Security staff and related infrastructure & High & 150,000 & 200,000 \\
\hline Technical support staff & High & 800,000 & 800,000 \\
\hline Functional staff & High & $1,150,000$ & $1,150,000$ \\
\hline Vendor/contract management staff & Medium & 50,000 & 50,000 \\
\hline Total & & $4,762,871$ & $6,731,715$ \\
\hline
\end{tabular}

\section{Discussion}

The study provides strong evidence to suggest that the cost-benefits of upgrading and maintaining an on-premise EHR in U-M Dent would be higher than adopting and operating a cloud-based EHR solution. The cloud-based EHR system performed similar to or better than the on-premise EHR for all three ECAR-TCO factors. As a result, U-M Dent decided to implement the cloud-based EHR. When fully implemented, the actual costs will be known.

The ability to effectively compare the costs of the two systems (on-premise vs. cloud) is essential to understanding the complete impact of IT costs in higher education environments and the cost shifts as we begin moving towards an increasing number of cloud services. ${ }^{20}$ In general, the usual costing and financial data for an IT project were considered insufficient to effectively inform the decision makers. ${ }^{2}$ By using the ECAR-TCO framework, U-M Dent was able to identify all pertinent (including hidden) costs of on-premise solutions. The ECAR-TCO framework aided the study as it went beyond the calculation of hard-dollar costs to account for qualitative factors that may not directly or easily translate to a number but address a strategic value of importance to higher education institutions. Including these factors and discussing them with the community provide an opportunity to identify potential risks and opportunities. The review process undertaken by U-M Dent has raised awareness of issues that might not be addressed if the assessment were limited to hard costs.

In response to criticism of the slow adoption of IT in the health sector, the poor safety record of care delivery systems, ${ }^{21}$ and the unacceptable lag time between discovery and translation into routine practice, the concept of a Learning Health System (LHS) has gained considerable momentum. ${ }^{22}$ An LHS, as described by the National Academy of Medicine (previously the Institute of Medicine), is characterized by five attributes: health-related relevant data are available for study; practice knowledge derived from these data is available; health improvements are routine and continuous processes; infrastructures enable 
the routine execution of multiple learning cycles; and activities are viewed as part of the culture. ${ }^{22,23}$ Cloudbased EHR systems provide easy access to data to a variety of users, while still adhering to strict security and access control mechanisms. Anecdotal research data collection is replaced with the availability of all data from routine patient care in cloud systems. Data use as a decision tool is shifted from furnishing infrequent clinical reports (in older on-premise EHR solutions) to real-time access to data combined with business intelligence data analysis tools for day-today operational decisions (in web-based cloud EHR systems). Furthermore, clinicians, patients, students, and administrators have access to the data they need and are permitted to interrogate data independent of physical location. Overall, cloud-based EHR systems inherently embrace the LHS and are in close alignment with leading national initiatives that will shape the future of the U.S. health care system.

\section{Foundational Factors}

Foundational factors (data sensitivity, business criticality, and vendor capability) are overarching factors and paramount to any IT solution. ${ }^{2}$ At U-M Dent, the cloud-based provider underwent a third-party security review and committed to ongoing security reviews including ISO 27001 certification. ${ }^{24}$ While on-premise applications may appear to be more secure because the institution owns and controls the services, they suffer more security incidents than cloud applications. ${ }^{25}$ Also, cloud application developers typically have dedicated security personnel, something few dental schools can afford, and are focused on security and governance such as obtaining ISO 27001 certification. ${ }^{26} \mathrm{~A}$ third-party security review examines the vendor, the development processes, and the product, whereas a traditional security review only tests the product itself. The objective is to make sure quality and security are "baked-in" from the beginning.

A dental school's EHR is generally considered its most critical IT application, and it needs to be reliable and responsive to the school's changing needs. When the IT infrastructure is fully managed by a vendor leveraging cloud services such as Amazon Web Services (AWS), it enables scalability at the touch of a button with a usage-only cost model. Cloud-based infrastructure scripts monitor capacity and performance, automatically recognizing when a threshold has been reached and automatically increasing capacity without a person ever having to intervene. ${ }^{27}$ One does not need a system administrator to add CPUs to a server, to purchase an additional server, or to create a virtual server. As cloud-based infrastructure is managed by the vendor, there is also no longer a need to have a database administrator to tune the database, design and implement database structures for customizations, and monitor performance. In addition, software for monitoring the infrastructure and staff time for reviewing reports and alerts is eliminated.

According to the ECAR-TCO framework, service providers' ability to deliver new functions is considered vendor/service provider capability, and how these functions are defined and prioritized is known as governance. ${ }^{2}$ EHR governance is about the dental school and the service provider agreeing upon a transparent process for decision making. For governance and, consequently, for the EHR to best meet the needs of the school, this process needs to ensure consistency and accountability through a strong vendor management process..$^{28}$

\section{Qualitative Factors}

This qualitative comparison found that a cloud EHR aligned more closely with the school's mission than the on-premise system. As a researchintensive school, U-M Dent innovates to further its research mission. Thus, the importance it placed on EHR standards, interoperability with other systems including other health records, and the innovative LHS are reflected in its priorities. ${ }^{29} \mathrm{U}-\mathrm{M}$ Dent intends to work with others to continue to innovate with its health record to improve overall health outcomes. U-M Dent has taken and continues to take seriously the notion of advancing dental health care through advanced innovation. Cloud-based technologies are already having an effect in medicine, ${ }^{30-32}$ so we have the obligation to evaluate the benefits of this technology for dentistry. It is through the precedent of such efforts that innovation has the potential of becoming widespread and, with that, resulting in global recognition of more effective dental treatment. For this reason, we at U-M Dent feel it is consistent with our mission to serve as an innovation hub and lead the profession.

\section{Quantitative Factors}

The summary of costs analyzed over two years includes the one-time costs of either implementing a cloud EHR or upgrading a traditional on-premise EHR. In all categories, the cloud EHR proved to be less costly than the on-premise EHR system. Of 
special note is that there are no hidden costs with a cloud system. EHRs are mission-critical systems, yet all too often disaster recovery functions are not fully accounted for in a cost analysis of an on-premise system; by contrast, it is built into a cloud system. Staffing is usually the most expensive component of any system. While additional staff are required to either upgrade an on-premise EHR or implement a cloud EHR (e.g., including integration with other systems, data migration, training, and report creation), the staffing costs for the cloud EHR are less than those for an on-premise EHR since it is a usage-only model. Of special note is that the ongoing costs of the cloud EHR eliminate the need for a database administrator and system administrator to support the cloud EHR.

\section{Limitations and Strengths}

This study had various limitations as well as strengths that warrant scrutiny. First, data for the study were collected across a two-year time frame. The data being analyzed were based on 2016 collections, and thus, it might not reflect current cost estimates. The estimates represent a snapshot in time, implying that if the same analysis were conducted a few years earlier or later, the results might differ. Therefore, we recommend that future studies take account of cost comparisons across a longer time frame (five to ten years). We also recommend, as additional schools adopt a cloud EHR, that costs from those institutions be included in this analysis.

Second, some factors that would potentially have a bearing on cloud-based solutions for dental health records have not been fully evaluated or measured. These include the future proof of effectiveness and the reputation missions of the institutions involved (i.e., aim to attract the best students or attain recruitment benefits). Aspects such as recruiting researchers who evaluate a workplace based on high-tech infrastructure or achieving the competitive advantage of being an innovator could have potential bearings. Also, we recognize that other institutions may have an IT cost model that differs from that at U-M Dent. These institutional differences cannot be accounted for in this analysis.

Finally, the analysis was only validated internally (by the U-M Dent team and stakeholders), as opposed to externally (such as an independent evaluating organization). This study represents an example for an applied enhanced (ECAR-TCO) framework in an academic dental setting for a purchase decision. By its very nature, TCO is an analysis meant to uncover all the lifetime costs that follow from owning certain kinds of assets. Therefore, TCO is sometimes called lifecycle cost analysis. TCO analysis is not a complete cost-benefit analysis because TCO tries to uncover ownership costs, but it does not consider other kinds of business benefits due to acquisitions, projects, or initiatives. While we have described some qualitative and quantitative factors, these are not exhaustive but merely attempts to address perhaps the most important issue for any academic dental institution wanting to explore the possibilities of converting to a cloud-based EHR solution: namely, its cost.

\section{Conclusion}

This study found that a cloud-based EHR system for U-M Dent offered significant cost savings and unique benefits that were not available with the traditional on-premise EHR solution. These findings suggest that a potential replication of a cloud-based EHR at another dental school will provide significant costs savings, although the numbers and percentages may vary. Cost savings, and more importantly, the intangible benefits showcase why higher education and private industry are moving towards cloud-based solutions. Being one of the first dental schools in the U.S. to undergo this transition, U-M Dent has made a strong case for the use of cloud-based EHR systems in other dental schools.

\section{Acknowledgments}

The authors would like to acknowledge Joe Kerr for his continuous wise advice and his feedback on the manuscript. Madhan Balasubramanian acknowledges support from a National Health and Medical Research Council (NHMRC) Sidney Sax Early Career Research Fellowship (Grant Number: 1121576). The contents are solely the responsibility of the authors and administering institution and do not reflect the views of any funding bodies.

\section{REFERENCES}

1. Bahga A, Madisetti VK. A cloud-based approach for interoperable electronic health records (EHRs). IEEE J Biomed Health Inform 2013;17(5):894-906.

2. ECAR-TCO Working Group. TCO for cloud services: a framework. April 24, 2015. At: library.educause.edu/ resources/2015/4/tco-for-cloud-services-a-framework. Accessed 2 Oct. 2017.

3. Muzra H, El-Masri S, eds. Cloud computing system for integrated electronic health records. Proceedings of the International Conference on Bioinformatics \& Computational Biology (BIOCOMP), 2012. 
4. Spallek H, Johnson L, Kerr J, Rankin D. Costs of health IT: beginning to understand the financial impact of a dental school EHR. J Dent Educ 2014;78(12):1542-51.

5. Stark PC, Kalenderian E, White JM, et al. Consortium for oral health-related informatics: improving dental research, education, and treatment. J Dent Educ 2010;74(10):1051-65.

6. Consortium for Oral Health Research and Informatics. Consortium members. 2017. At: cohri.org/?page_id=13. Accessed 2 Oct. 2017.

7. White JM, Kalenderian E, Stark PC, et al. Evaluating a dental diagnostic terminology in an electronic health record. J Dent Educ 2011;75(5):605-15.

8. Schleyer TK, Thyvalikakath TP, Spallek H, et al. From information technology to informatics: the information revolution in dental education. J Dent Educ 2012; 76(1):142-53.

9. Ferraiolo D, Spivakovsky S. Do EHRs affect students' abilities to develop critical thinking skills? J Acad Distinguished Educators 2016;4(1):7-11.

10. Polverini P, Krebsbach P. Research and discovery science and the future of dental education and practice. J Dent Educ 2017;81(9 Suppl):eS97-107.

11. Walji M. The EHR of the future: supportive, collaborative, and barely noticeable. J Acad Distinguished Educators 2016;4(1):12-9.

12. Walji MF, Kalenderian E, Tran D, et al. Detection and characterization of usability problems in structured data entry interfaces in dentistry. Int J Med Inform 2013;82(2):128-38.

13. Emrick JJ, Gullard A. Integrating research into dental student training. J Dent Res 2013;92(12):1053-5.

14. Guven Y, Uysal O. The importance of student research projects in dental education. Eur J Dent Educ 2011;15(2):90-7.

15. Nalliah RP, Lee MK, Da Silva JD, Allareddy V. Impact of a research requirement in a dental school curriculum. J Dent Educ 2014;78(10):1364-71.

16. Hillestad R, Bigelow J, Bower A, et al. Can electronic medical record systems transform health care? Potential health benefits, savings, and costs. Health Aff 2005;24(5):1103-17.

17. University of Michigan. Dentistry schools move forward with new health record system. 2017. At: www.dent. umich.edu/news/2017/01/31/dentistry-schools-moveforward-new-health-record-system\#sthash.u9GejFVW. dpbs. Accessed 2 Oct. 2017.

18. EDUCAUSE. About EDUCAUSE. 2017. At: www.educause.edu/about. Accessed 2 Oct. 2017.
19. EDUCAUSE Centre for Analysis and Reasarch. ECAR working groups. 2017. At: www.educause.edu/ecar/ecarworking-groups. Accessed 2 Oct. 2017.

20. Bedrossian A, Bergsmark B, Carozzoni B, et al. Cloud strategy for higher education: building a common solution. Louisville, CO: Cloud Solutions, 2014.

21. Foley T, Vale L. What role for learning health systems in quality improvement within health care providers? Learn Health Syst 2017;1(e10025).

22. Smith M, Saunders R, Stuckhardt L, McGinnis JM, eds. Best care at lower cost: the path towards continuous learning health care in America. An Institute of Medicine Report. Washington, DC: National Academies Press, 2013.

23. Olsen L, Aisner D, McGinnis J. Institute of Medicine roundtable on evidence-based medicine: LHS workshop summary. Washington, DC: National Academies Press, 2007.

24. International Standards Organization. ISO/IEC 27002: 2013 information technology, security techniques, code of practice for information security controls. $2^{\text {nd }}$ ed. 2013. At: iso27001 security.com $/ \mathrm{html} / 27002 . \mathrm{html}$. Accessed 29 Jan. 2018.

25. Alert Logic. Cloud security report. 2017. At: www. alertlogic.com/resources/cloud-security-report-2017/. Accessed 30 Jan. 2018.

26. Sullivan D. Prove the security of cloud computing in your organization. 2016. At: searchcloudcomputing.techtarget. com/tip/How-to-squash-security-concerns-with-cloudcomputing. Accessed 30 Jan. 2018.

27. Amazon. AWS auto scaling. 2017. At: aws.amazon.com/ autoscaling/. Accessed 30 Jan. 2018.

28. Weill P, Ross J. IT governance: how top performers manage IT decision rights for superior results. Boston: Harvard Business Review, 2004.

29. Friedman C, Wong AH, Blumenthal D. Achieving a nationwide learning health system. Sci Transl Med 2010;2(57):1-3.

30. Ahmadi M, Aslani N. Capabilities and advantages of cloud computing in the implementation of electronic health record. Acta Inform Med 2018;26(1):24-8.

31. Mayo RC 3rd, Pearson KL, Avrin DE, Leung JW. The economic and social value of an image exchange network: a case for the cloud. J Am Coll Radiol 2017;14(1):130-4.

32. Feeney JM, Montgomery SC, Wolf L, et al. Cost savings associated with the adoption of a cloud computing data transfer system for trauma patients. Conn Med 2016;80(7):389-92. 